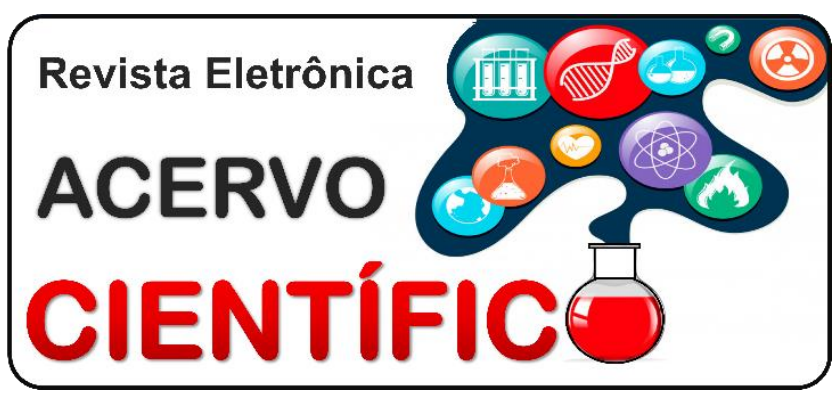

\section{REVISÃO BIBLIOGRÁFICA}

Recebido em: 9/2020

Aceito em: 10/2020

Publicado em: 11/2020

\title{
Como os tratamentos alternativos e complementares para a esclerose múltipla contribuem para a qualidade de vida dos pacientes portadores da doença
}

\author{
How alternative and complementary treatments for multiple sclerosis contribute to the \\ quality of life of patients with the disease
}

Cómo contribuyen los tratamientos alternativos y complementarios para la esclerosis múltiple a la calidad de vida de los pacientes con la enfermedad

Thaynã Varges Gomes ${ }^{1 *}$, Stéfane Coelho dos Santos ${ }^{1}$, Carolina Ketley Pinheiro Rodrigues ${ }^{1}$, Vanessa Loures Rossinol ${ }^{1}$, Brenda Gomes Vieira ${ }^{1}$, Gabriela Brito Santos ${ }^{1}$, Sara Cristina Barbosa Ribeiro ${ }^{1}$, Thaís Vieira da Silva1.

\begin{abstract}
Resumo: Esse artigo buscou discorrer sobre tratamentos alternativos e complementares para Esclerose Múltipla e como interferem na qualidade de vida dos pacientes com a doença, considerando benefícios e/ou malefícios dos procedimentos. Trata-se de uma revisão bibliográfica fundamentada em pesquisa de dados online em plataformas como Medical Literature Analysis and Retrievel System Online (Medline), Public/ Publisher Medline (Pubmed), Scientific Electronic Library Online (Scielo) e Literatura Latino-americana e do Caribe em Ciências da Saúde (LILACS). Foram escolhidos vinte e quatro artigos, tendo como critério de seleção o período de publicação dos artigos de 2002 a 2020, sendo assim, foram selecionados artigos com ênfase nos tratamentos não convencionais para Esclerose Múltipla e qualidade de vida dos pacientes com a doença. Tal revisão apresenta como opções de tratamentos alternativos e complementares a utilização de vitamina $\mathrm{D}$, fisioterapia, terapia ocupacional e perspectivas futuras como o Transplante Autólogo de Células Tronco e o uso de Cannabis, visto que quando usados no momento certo, de forma correta e com profissionais competentes podem beneficiar os pacientes, seja juntamente com o tratamento tradicional ou quando esse já não traz resultados satisfatórios. Desse modo, visam a promoção de bem-estar e autonomia ao indivíduo.
\end{abstract}

Palavras-chave: Esclerose múltipla, Qualidade de vida, Tratamentos.

Abstract: This article sought to discuss alternative and complementary treatments for Multiple Sclerosis and how they affect the quality of life of patients with the disease, considering the benefits and/ or harms of the procedures. This is a bibliographic review based on online data research on platforms such as Medical Literature Analysis and Retrievel System Online (Medline), Public / Publisher Medline (Pubmed), Scientific Electronic Library Online (Scielo) and Latin American and Caribbean Literature in Health Sciences (LILACS). Twenty-four articles were chosen, having as selection criteria the period of publication of articles from 2002 to 2020, therefore, articles were selected with an emphasis on unconventional treatments for Multiple Sclerosis

${ }^{1}$ Centro Universitário de Caratinga (UNEC), Caratinga - MG. *E-mail: thaynavarges@outlook.com 
and quality of life of patients with the disease. Such review presents as alternative and complementary treatment options the use of vitamin D, physiotherapy, occupational therapy and future perspectives such as Autologous Stem Cell Transplantation and the use of Cannabis, since when used at the right time, correctly and with professionals Competent professionals can benefit patients, either together with traditional treatment or when it no longer brings satisfactory results. Thus, they aim to promote the individual's well-being and autonomy.

Keywords: Multiple sclerosis, Quality of life, Treatments.

Resumen: Este artículo busca discutir los tratamientos alternativos y complementarios para la Esclerosis Múltiple y cómo afectar la calidad de vida de los pacientes con la enfermedad, considerando los beneficios y/o los procedimientos. Se trata de una revisión bibliográfica basada en búsquedas de datos en línea en plataformas como Medical Literature Analysis and Retrievel System Online (Medline), Public / Publisher Medline (Pubmed), Scientific Electronic Library Online (Scielo) y Latin American and Caribbean Literature. en Ciencias de la Salud (LILACS). Si se seleccionaron artículos, se eligió el período de selección de los artículos de 2015 a 2020 como criterio para seleccionar artículos con énfasis en el tratamiento no convencional de la esclerosis múltiple y la calidad de vida de los pacientes con la enfermedad. Esta revisión presenta opciones de tratamiento alternativas y complementarias para el uso de vitamina $D$, fisioterapia, terapia ocupacional y perspectivas de futuro como el Autotrasplante de Células Madre y el uso de Cannabis, que cuando se usa correctamente, con los profesionales adecuados. Profesionales competentes puede beneficiar a los pacientes, por lo que va de la mano del tratamiento tradicional o cuando no da resultados satisfactorios. Tienen como objetivo promover el bienestar y la autonomía del individuo.

Palabras clave: Esclerosis múltiple, Calidad de vida, tratos.

\section{INTRODUÇÃO}

A Esclerose Múltipla (EM) é uma doença inflamatória, crônica e progressiva que acomete, principalmente, adultos jovens no auge da vida produtiva. Nessa doença, as bainhas de mielina, membranas que envolvem as fibras do Sistema Nervoso Central (SNC), cuja função é permitir que o impulso elétrico ocorra de forma saltatória, rápida e eficaz, são lesionadas, levando a múltiplos focos de desmielinização. A doença causa, portanto, incapacidade neurológica permanente a longo prazo, além de diversas alterações psicológicas, déficits cognitivos e fadiga, levando ao comprometimento da qualidade de vida dos portadores e, consequentemente, na vivência do paciente nos âmbitos social, pessoal e profissional. De acordo com a periodicidade dos surtos, a EM é classificada em: Esclerose Múltipla Remitente Recorrente (EMRR), Esclerose Múltipla Primária Progressiva (EMPP), Esclerose Múltipla Secundária Progressiva (EMSP) e Esclerose Múltipla Progressivo-Recorrente (EMPR) (ALVES BCA, et al., 2014; NOVAIS NGP, et al., 2014).

A crescente preocupação com a qualidade de vida relacionada à saúde da pessoa não preza apenas pela eliminação dos sintomas da doença, mas também pela redução da mortalidade e o aumento da esperança média de vida. Dessa forma possui um papel fundamental para os profissionais de saúde no que tange à escolha de melhores opções terapêuticas (RODRIGUES CCP e HENRIQUES DMF, 2018).

No momento, preferencialmente, a escolha pelos tratamentos de melhor prognóstico para a EM tem se tornado um desafio para os neurologistas. Isso porque a alta complexidade da patologia ocorre por interações entre diferentes genes e influência de fatores ambientais que ainda não estão totalmente elucidados. Essa complexidade está ligada ao pleiomorfismo desta patologia, que causa variedade fenotípica e leva a diferentes reações a intervenções e a medicamentos. Estudos já realizados descobriram muitos tratamentos para o subtipo Emitente Recorrente da EM com um impacto favorável à doença, porém nenhuma Terapia Modificadora de Doença (DMT) está atualmente aprovada para o curso clínico primário progressivo da EM (GAJOFATTO A, et al., 2016). 
A disposição de medicamentos específicos para a EM no Brasil segue o protocolo clínico para recomendações terapêuticas do Ministério da Saúde que estabelece critérios seguros baseados em qualidade técnica e científica a respeito de condutas diagnósticas e terapêuticas que devem ser adotadas por profissionais da saúde, porém, apesar de atender às obrigações regulatórias do Estado, pode ser considerado inflexível. O protocolo, por devida necessidade, se baseia em diretrizes farmacoeconômicas e gera certa limitação a respeito do tratamento que seria mais satisfatório para cada paciente, pois ainda não leva totalmente em consideração os atuais avanços no conhecimento da doença a respeito da heterogeneidade individual, da complexidade da patologia e nem das diferentes respostas terapêuticas que cada paciente pode manifestar, o que, dificulta o avanço para o tratamento da Esclerose Múltipla (COMINI-FROTA RE, et. al., 2016).

Ser portador de uma doença crônica, como a Esclerose Múltipla que gera uma gama de manifestações clínicas, pode causar grandes sentimentos de frustração, estresse e ansiedade. É necessário intervir precocemente, dando importância a todos os sinais e sintomas que possam gerar dependência e desconforto, com o objetivo de minimizar as consequências negativas da terapêutica adotada (RODRIGUES CCP e HENRIQUES DMF, 2018).

Os pacientes demandam de um cuidado sistematizado e humanizado, sendo importante a implementação de métodos que promovam melhor adaptação ao contexto heterogêneo da doença e redução dos impactos negativos na qualidade de vida desses. O cenário brasileiro atual, em relação às opções terapêuticas que disponham de tais características, encontra-se, de certa forma, limitado, o que justifica o estudo de estratégias e tratamentos inovadores.

Nesse viés, determina-se como objetivo do presente trabalho realizar uma revisão narrativa acerca de como os tratamentos alternativos e complementares para esclerose múltipla podem afetar a qualidade de vida de pacientes portadores da doença, levando em consideração seus prós e contras.

\section{REVISÃO BIBLIOGRÁFICA}

\section{Tratamentos alternativos e complementares para a esclerose múltipla}

Sendo a EM uma doença progressiva e, ainda, sem cura, é evidente que as limitações por ela causadas trazem impactos negativos relevantes à qualidade de vida dos pacientes portadores. Soma-se a isso, os possíveis efeitos colaterais da medicação, impactando, também, de forma desfavorável, na própria percepção de bem-estar do indivíduo. Uma vez que essa cronicidade permite que múltiplos focos do sistema nervoso sejam afetados, mesmo que haja uma tentativa compensatória do corpo para suprir as perdas de sensibilidade e função, isso não promove a retomada da capacidade física e cognitiva de anteriormente. Com isso, surgem os sintomas como fraqueza e espasticidade tão característicos da doença (SILVA MCN e CAVALCANTI DBA, 2019). Com o intuito de fazer uma abordagem dos tratamentos não convencionais, será apresentado de uma forma geral os seguintes tratamentos alternativos e complementares: tratamento para esclerose múltipla com utilização de vitamina $\mathrm{D}$, fisioterapia, terapia ocupacional e perspectivas futuras.

\section{Tratamento para Esclerose múltipla com utilização de Vitamina D}

A vitamina $D$ é sintetizada na pele por meio de um precursor denominado 7-desidrocolesterol, que pela clivagem fotoquímica, tem como produto a vitamina D3. Ademais, a absorção da vitamina $D$ ocorre no intestino, sua maioria encontra-se na forma circulante $(25(\mathrm{OH}))$ e a forma ativa nas células do túbulo contorcido proximal, na região renal. $O$ principal papel da vitamina $D$ é, além da absorção de cálcio no intestino, o controle da retirada do íon a partir do tecido ósseo e a elevação da reabsorção renal de cálcio no túbulo distal. Há também, como outra função fundamental, a comunicação com o sistema imunitário em que se têm receptores de vitamina D em vários tecidos corporais (MARQUES CDL, et al., 2010).

Nos estudos de Deseilligny CP e Souberbielle JC (2017), foi demonstrado a desigualdade geográfica da radiação solar. Resultante disso, as pessoas que vivem em regiões subtropicais e tropicais são mais acessíveis aos raios ultravioleta $B$, que é um fator fotoquímico imprescindível para a síntese da vitamina $D$. 
Contraditoriamente, pessoas que vivem em regiões de clima predominantemente temperado no hemisfério norte recebem poucos raios solares tornando-se mais susceptíveis a desenvolver EM. Nesse contexto, notase que a permanência sem exposição solar por longos períodos é um fator para a hipovitaminose da vitamina D, o que pode se acentuar em locais que já recebem menores incidências. Também, é importante atentar-se para a pouca exposição solar na gravidez e na infância, já que pode acarretar influências genéticas, levando o indivíduo a tornar-se mais propício a desenvolver EM (BRUM DG, et al., 2014; LUCAS RM, et al., 2015).

Por outro lado, deve-se atentar ao tempo da exposição solar e fazer o uso de proteção, evitando os efeitos negativos da exposição exagerada, como o câncer de pele. Outrossim, é necessário que haja um acompanhamento profissional específico para que não aconteça intoxicações por vitamina $D$ que podem ter como sintomas náuseas e vômito, anorexia, dor abdominal, polidipsia, poliúria, desidratação, nefrolitíase, nefrocalcinose dentre outros (RIBEIRO GG, et al., 2019; BRUM DG, et al., 2014).

Além disso, pressupõe-se que, a importância da influência da vitamina $D$, no controle do sistema imunitário, resulta da ocorrência do bloqueio exacerbado de células pró-inflamatórias e da produção de citocinas inflamatórias. Nesse viés, a interação da vitamina $D$ com as células do sistema imunológico acaba promovendo a diminuição do processo inflamatório e aumenta as citocinas anti-inflamatória (TGF- $\beta 1$ ), após uma suplementação em um prazo de, no mínimo, 6 meses (RIBEIRO GG, et al., 2019).

De acordo com a Academia Brasileira de Neurologia devem ser consideradas algumas orientações para a utilização da vitamina $D$ nos portadores da EM, como a realização da dosagem sérica, para efetuar a supervisão da suplementação antes e depois do tratamento, manter a suplementação entre doses de $40 \mathrm{ng} / \mathrm{ml}$ e $100 \mathrm{ng} / \mathrm{ml}$ e não usar a Vitamina D como monoterapia no tratamento da Esclerose múltipla. Ademais, a vitamina $D$ contém baixa toxicidade, menos efeitos adversos, seu custo é mais vantajoso, comparando-se com outros imunossupressores comercializados (RIBEIRO GG, et al., 2019; BOAS V, et al., 2016).

Apesar de ser importante se atentar quanto ao tempo de exposição solar e quanto aos riscos de intoxicação que níveis elevados podem causar, a utilização da vitamina $D$ pode ser promissora para a EM. Por sua aparente influência no sistema imune, como moduladora, além de ação sobre o metabolismo do cálcio contribuições nessas funções seriam relevantes (ASHIERO A, et al., 2010).

Observa-se que estudos realizados já revelam a eficiência e segurança nos resultados desse tratamento, sendo promissor para a aplicação da vitamina D como monodroga ou juntamente com tratamentos atuais, porém é considerado que outros estudos ainda devem ser feitos. Assim sendo, pode-se possibilitar uma farmacologia segura, redução dos gastos com os tratamentos da EM, possivelmente alcançar bons resultados e, dessa forma, aumentar os índices de qualidade de vida para esses pacientes (VILAS BOAS RB e ROCHA MS, 2016).

\section{Fisioterapia para o tratamento da esclerose múltipla}

Apesar do uso de várias drogas para o tratamento da esclerose múltipla na tentativa de alterar o seu curso, essas drogas não são o bastante para recuperar as funções que já foram perdidas em decorrência da doença. Portanto, é de suma importância que a medicação seja combinada com a prática da fisioterapia, uma vez que a fisioterapia contém técnicas e exercícios que podem aperfeiçoar a mobilidade dos portadores da esclerose múltipla e assim aprimorar as atividades diárias (VARGAS AL, et al., 2002).

Dentre os sintomas da EM a fadiga é um dos mais frequentes, podendo ser muito debilitante. Dessa forma, atividades simples do cotidiano podem se tornar mais cansativas e difíceis de serem realizadas. Portanto, exercícios moderados que conservam energia, uso de dispositivos de ajuda, simplificação do trabalho e resfriamento são necessários, já que podem ser eficazes para amenizar a clínica de fadiga do paciente. $\mathrm{Na}$ fisioterapia as limitações impostas pela doença são trabalhadas para que seja melhorada a capacidade funcional e como consequência possibilitar a qualidade de vida e precaver complicações debilitantes (RODRIGUES IF, et al., 2008).

No estudo realizado por Rodrigues IF, et al. (2008) pode-se concluir que, apesar dos indivíduos apresentarem risco para quedas e estarem abaixo do nível esperado para qualidade de vida, a fisioterapia 
proporcionou melhora no equilíbrio e na funcionalidade. Ademais, segundo Pereira GC, et al. (2012) programas de fisioterapia que utilizam alongamento muscular e exercícios ativos livres demonstrou que pessoas expostas a programas intensos de fisioterapia tinham deterioração significativamente menor se comparado aqueles que recebiam menos tratamento. As atividades funcionais, de equilíbrio e da vida diária também melhoraram significativamente no grupo que recebeu mais tratamento.

Vale ressaltar que profissionais desta área devem estar preparados para diversas situações, sejam elas profissionais, pessoais e psicológicas, pois muitas vezes, durante $\mathrm{o}$ atendimento, os pacientes discutem sobre suas questões pessoais, suas angústias e inseguranças. Portanto, é necessário que estes profissionais estejam prontos para essas situações e procurem a interação com uma equipe multidisciplinar, principalmente com psicólogo, com o intuito de ser oferecido um tratamento adequado e humanizado aos pacientes. Ademais, indivíduos que se encontram em condições clínicas graves decorrentes de condições neurológicas, como a esclerose múltipla, têm contato com o fisioterapeuta nas unidades de terapia intensiva, tendo, portanto, o risco de vida. Por isso, é muito importante que o profissional esteja apto na parte técnica e no âmbito ético para enfrentar este momento difícil e delicado (BIM CR, et al., 2007).

Apesar das necessidades serem individuais, ou seja, para cada paciente as necessidades são variáveis de acordo com o nível de acometimento da doença, alguns objetivos gerais são comuns ao tratamento de portadores da EM. Alguns dos objetivos são; a melhora na qualidade dos padrões de movimento, incentivo no desenvolvimento de estratégias de movimento, melhoria na força muscular, melhoria na coordenação motora, redução das anormalidades de tônus muscular, melhora na estabilidade postural, entre outros. A definição dos objetivos e escolha do tratamento devem ser planejadas de forma cautelosa de acordo com as necessidades individuais e da capacidade do paciente. Sempre deve ser considerado o que o paciente pode alcançar, não excedendo seus graus de dificuldade (VARGAS AL, et al., 2002).

Nesse viés, fica implícito a importância da associação do tratamento utilizado pelo paciente com a fisioterapia para que melhorias na qualidade de vida ocorram nos âmbitos individual e familiar dos indivíduos. Dessa maneira, problemas emocionais como estresse, depressão e angústia advindos do fato de ser portador de uma doença crônica podem ser minimizados. Ademais, além de uma equipe multidisciplinar no acompanhamento é de suma importância a ajuda emocional por parte da família e amigos para que, dessa forma, o tratamento alcance melhores índices de satisfação de ambas as partes.

\section{Terapia ocupacional}

Os cuidados da Terapia Ocupacional na Esclerose Múltipla apresentam-se como uma estratégia de desenvolvimento do autocuidado, melhora da apropriação corporal e da autopercepção do indivíduo, visando promoção de saúde, bem-estar, reabilitação, melhora das funções corporais, superação de limites e prevenção de incapacidades esperadas na doença. A promoção da consciência corporal nesses pacientes é importante para que haja o estímulo e o equilíbrio de funções orgânicas, físicas e psíquicas por meio de percepções de como o corpo funciona. Isso possibilita a melhora de funções básicas diárias, atingindo o principal objetivo do tratamento que inclui maior participação social e qualidade de vida do paciente no meio em que está inserido (PIMENTEL PP e TOLDRÁ RC, 2017a).

Conforme estudos de Campos LAB e Toldrá RC, (2019); o acompanhamento da Terapia Ocupacional auxilia na melhora dos sintomas, utilizando métodos e técnicas como: Conservação de Energia Corporal para reduzir o impacto provocado pela fadiga; Estratégias de Autogeração, ou seja, produção de palavras e conteúdos pelo próprio paciente, para a reabilitação da memória, desempenho das atividades da vida cotidiana, satisfação pessoal e melhora da depressão; Aplicação de Programas Domiciliares para estimular a independência, segurança, destreza manual e avanço nas funções diárias, além de ajudar a prevenir quedas por meio de exercícios de força muscular e alongamento; Utilização de Recursos Tecnológicos para aprimoramento de habilidades, eliminação de barreiras para realização de exercícios físicos e lazer; Contando também com Programas em Grupos baseados na técnica do Self-Healing para que haja compartilhamento de experiências entre pacientes, mostrando eficácia na redução de prejuízos cognitivos, equilíbrio e promoção do autoconhecimento. 
Não foram encontradas contraindicações referentes ao tratamento, sendo importante destacar que o acompanhamento é feito de forma personalizada, atendendo às necessidades terapêuticas de caráter individual. Além disso, é preciso que se construa um bom vínculo profissional para que haja o compartilhamento de opiniões e assegure o respeito à autonomia do usuário e seus familiares na tomada de decisões responsáveis. Sendo assim, a estabilidade dessa relação é imprescindível para que alcance resultados satisfatórios, garanta uma boa adesão ao tratamento, contribuindo na prevenção de comportamentos e atitudes que prejudiquem a saúde (PIMENTEL PP e TOLDRÁ RC, 2017b).

\section{Perspectivas futuras de tratamento}

Além de existirem os tratamentos tradicionais existem também os alternativos e complementares. Devido à variedade sintomatológica, a terapêutica de escolha deve ser a que melhor supre as necessidades do paciente. Diante do quadro e estágio da doença, além da adesão a uma medicação tradicional, pode-se orientar a alternativa e, também, a prática de atividades físicas e encaminhamento nutricional, que podem trazer melhora ao prognóstico. Como perspectivas futuras de tratamento, encontra-se o transplante autólogo de células tronco (TACT) e o uso de Cannabis. O primeiro pode ser utilizado em pacientes sem resposta aos tratamentos tradicionais. Já em relação ao segundo, sua funcionalidade está mais relacionada à redução de espasmos (ALVES BCA, et al., 2014).

Quanto aos sintomas de dor neuropática seja crônica, seja aguda, existem, na não responsividade à terapêutica tradicional, os tratamentos não convencionais que utilizam canabinóides, possuidores de ação sedativa. Além desse poder analgésico, possuem influência, também, sobre a ansiedade. Sendo bem mais potentes do que a morfina e livres de seus efeitos colaterais, a regulamentação quanto ao seu cultivo no Brasil ainda é proibitiva (RABELO AQ, et al., 2019). O uso de canabinóides é uma possibilidade terapêutica complementar eficaz em alguns casos, porém ainda necessita de mais análises e sob um maior período de tempo. É necessário, contudo, que antes da adoção dessa escolha, a sintomatologia seja observada a fim de evitar o surgimento de novos problemas. Essa opção de tratamento pode ser promissora para as manifestações de espasmos e dor. Já quanto à disfunção vesical e aos tremores não foram demonstrados sinais de melhora (BRUCKI SMD, et al., 2015).

Com relação ao transplante de células-tronco hematopoéticas (TCTH), os efeitos promissores para a EM a colocam como indicada ao tratamento. Tendo como foco o paciente e sabendo da menor expectativa de vida, o investimento em terapêuticas inovadoras é de grande importância. O TCTH, no entanto, é para um grupo específico, como os que não respondem ao convencional e os portadores da "forma maligna" da doença. Ademais, deve ser utilizado de forma precoce e na fase inflamatória da EM (VOLTARELLI JC, et al., 2009).

\section{CONSIDERAÇÕES FINAIS}

É visível que os tratamentos alternativos e complementares para pacientes portadores da Esclerose múltipla são benéficos se realizados da maneira adequada, por uma equipe profissional treinada e no estágio correto da doença. Estudos realizados sobre o assunto estão evoluindo gradativamente para que novas descobertas sejam realizadas com o intuito de ampliar os conhecimentos e, consequentemente, apresentar melhores prognósticos aos indivíduos portadores. Porém, vale ressaltar que a realização dos tratamentos alternativos e complementares não dispensa a utilização dos tratamentos convencionais. Portanto, concluise que seguindo todas as instruções para realização dos tratamentos, os pacientes poderão ter grandes benefícios e, dessa forma, induzir a melhora na qualidade de vida.

\section{REFERÊNCIAS}

1. ALVES BCA, et al. Esclerose múltipla: revisão dos principais tratamentos da doença. Saúde E Meio Ambiente: Revista Interdisciplinar, 2014; 3(2): 19-34.

2. ASCHERIO, et al. Vitamina D e esclerose múltipla. Reveja, 2010; 9: 599-612. 
3. BIM CR, et al. Fisioterapia no enfrentamento de perdas em pacientes com doenças neurológicas. Fisioterapia em movimento, 2007; 20(3): 71-78.

4. BRUCKI SMD, et al. Canabinoides e seu uso em neurologia - Academia Brasileira de Neurologia. Arq. NeuroPsiquiatr, 2015; 73(4): 371-374.

5. BRUM DG, et al. Suplementação e uso terapêutico de vitamina D nos pacientes com esclerose múltipla: Consenso do Departamento Científico de Neuroimunologia da Academia Brasileira de Neurologia. Arq Neuropsiquiatr, 2014; 72(2): III-VII.

6. CAMPOS LAB, TOLDRÁ RC. Intervenções de Terapia Ocupacional com pessoas com esclerose múltipla: revisão integrativa da literatura. Cadernos Brasileiros de Terapia Ocupacional, 2019; 27(4): 885-897.

7. COMINI-FROTA RE, et. al. Orientações para tratamento da esclerose múltipla no Brasil: Consenso do Departamento Científico de Neuroimunologia da Academia Brasileira de Neurologia. Arq. Neuro-Psiquiatr, 2017; 75(1): 57-65.

8. DESEILLIGNY CP, SOUBERBIELLE JC. Vitamin D and multiple sclerosis: An update. Multiple Sclerosis and Related Disorders, 2017;14: 35-45.

9. GAJOFATTO A, et. al. Primary progressive multiple sclerosis: current therapeutic strategies and future perspectives. Expert Review of Neurotherapeutics, 2016; 17(4): 393-406.

10. LUCAS RM, et al. Ultraviolet radiation, vitamin D and multiple sclerosis. Neurodegener. Dis. Manag., 2015; 5(5): 413424.

11. MARQUES CDL, et al. A importância dos níveis de vitamina D nas doenças autoimunes. Rev. Bras. Reumatol., 2010; 50(1): 67-80.

12. NOVAIS NGP, et. al. The effects of progressive muscular relaxation as a nursing procedure used for those who suffer from stress due to multiple sclerosis. Rev. Latino-Am. Enfermagem, 2016; 24.

13. PEREIRA GC, et al. Combinações de Técnicas de Fisioterapia no Tratamento de Pacientes com Esclerose Múltipla: Série de Casos. Rev Neurocienc., 2012; 20(4): 494-504.

14. PIMENTEL PP, TOLDRÁ RC. Desenvolvimento de manual para orientações básicas do dia a dia para pessoas com esclerose múltipla. Cad. Bras. Ter. Ocup., 2017; 25(1): 67-74.

15. PIMENTEL PP, TOLDRÁ RC. Método self-healing como estratégia de promoção à saúde e reabilitação de pessoas com esclerose múltipla no contexto da terapia ocupacional. Cad. Bras. Ter. Ocup., 2017; 25(3): 565-573.

16. RABELO AQ, et al. Uso terapêutico de canabinóides na esclerose múltipla. Ensaios USF, 2019; 3(1): $12-26$.

17. RIBEIRO GG, et al. Suplementação de vitamina D em pacientes com esclerose múltipla. Revista Medicina e Saúde, 2019; 2(4): 111-120.

18. RODRIGUES CCP, HENRIQUES DMF. Qualidade de vida da pessoa com esclerose múltipla e disfunção urinária: validação do Qualiveen. Revista de Enfermagem Referência, 2018; IV(18): 103-112.

19. RODRIGUES IF, et al. Avaliação da fisioterapia sobre o equilíbrio e a qualidade de vida em pacientes com esclerose múltipla. Rev. Neurocienc, 2008; 16(4): 269-274.

20. SILVA MCN, CAVALCANTI DBA. Avaliação da qualidade de vida em portadores de esclerose múltipla: impacto da fadiga, ansiedade e depressão. Fisioterapia e Pesquisa, 2019; 26(4): 339-345.

21. VARGAS AL, et al. Exercícios terapêuticos para portadores de Esclerose Múltipla com déficit de coordenação motora e equilíbrio. Fisioterapia Brasil, 2002; 3(3): 151-156.

22. VILAS BOAS RB, ROCHA MS. A importância da vitamina D na Esclerose Múltipla. Revista Acadêmica Oswaldo Cruz, 2016; 3(12).

23. VOLTARELLI JC, et al. Consenso Brasileiro para Transplante de Células-Tronco Hematopoéticas para Tratamento de Doenças Autoimune. Revista Brasileira de Hematologia e Hemoterapia, 2010;.32(1): 125-135. 